PROCEEDINGS OF THE

AMERICAN MATHEMATICAL SOCIETY

Volume 89, Number 1. September 1983

\title{
TRANSFERS, CENTERS, AND GROUP COHOMOLOGY ${ }^{1}$
}

\author{
DANIEL H. GOTTLIEB
}

\begin{abstract}
The transfer for fibrations is shown to exist for fibres with finitely generated total integral homology groups. This improvement is applied to cohomology of groups.
\end{abstract}

1. Introduction. The transfer for Hurewicz fibrations in [BG] was constructed for a fibration whose fibre $F$ is homotopy equivalent to a finite $\mathrm{CW}$-complex. In fact, it holds when $F$ has a finitely generated total integral homology group. That is when $H_{*}(F ; \mathbf{Z})$ is finitely generated. A corollary involving $\omega: X^{X} \rightarrow X$ also holds for finitely generated $H_{*}(X ; \mathbf{Z})$. These results are stated in the context of (co)homology of groups, where the improvement is particularly useful. There are transfer homomorphisms for surjections $G \stackrel{\rho}{\rightarrow} K$ whose kernels have finitely generated homology. The result concerning $\omega$ translates into a theorem about the centers of groups $G$ with finitely generated $H_{*}(G ; \mathbf{Z})$.

We apply these results to obtain the following two theorems. We denote by $[H, G]$ the group generated by commutators $h g h^{-1} g^{-1}$ such that $h \in H$ and $g \in G$, and by $\chi(H)$ the Euler-Poincare number of the group $H$. That is

$$
\chi(H)=\sum(-1)^{i} \operatorname{rank}\left(H_{i}(H ; \mathbf{Z})\right) .
$$

THEOREM 4. Let $H$ be a normal subgroup of $G$ such that $H_{*}(H ; \mathbf{Z})$ is finitely generated. Then $h^{\chi(H)} \in[H, G]$ for all $h \in H \cap[G, G]$.

TheOREM 5. Let $H_{*}(H ; \mathbf{Z})$ be finitely generated. Let $C$ be a central subgroup of $H$. Then $c^{x(H)} \in[H, H]$ for all $c \in C$.

We also apply the transfer to the case of central extensions of free abelian groups of finite rank.

2. Stably finite complexes. Spanier-Whitehead duality is always exposed in the literature as holding for finite complexes. But it is a triviality to observe that it works just as well for stably finite complexes. By a stably finite complex we mean a $\mathrm{CW}$-complex $X$ for which some suspension $S^{k} X$ is homotopy equivalent to a finite complex.

In fact a duality map is defined by $\mu: X \wedge X^{*} \rightarrow S^{n}$ for some $n$ such that the homomorphism $H_{q}(X ; \mathbf{Z}) \rightarrow H^{n-q}\left(X^{*} ; \mathbf{Z}\right)$ given by the slant product $\left(\mu^{*}\left[S^{n}\right] / \cdot\right)$ is

Received by the editors September 1, 1982.

1980 Mathematics Subject Classification. Primary 55R05, 20 S05.

'This research was supported by a grant from the National Science Foundation.

(C)1983 American Mathematical Society $0002-9939 / 82 / 0000-1430 / \$ 02.75$ 
an isomorphism. Spanier showed that for every finite complex $X$ and some $n$ there is a duality map $\mu: X \wedge X^{*} \rightarrow S^{n}$, and $X^{*}$ is called the dual of $X$. It is then immediate that if $X$ is stably finite, there must exist a duality map $\mu: X \wedge X^{*} \rightarrow S^{n}$.

In [BG] the concept of Spanier-Whitehead duality is extended to Hurewicz fibrations over $B$ with well-based cross-sections. A duality map is a fibre preserving map from $B \times S^{n} \rightarrow E \wedge_{B} \hat{E}$ (where $E$ and $\hat{E}$ are the total space of fibrations with specified cross-section and $\wedge_{B}$ means reduced fibrewise join) such that $S^{n} \rightarrow F_{b} \wedge \hat{F}_{b}$ is a duality map for every fibre $F_{b}$ and $\hat{F}_{b}$ of $E$ and $\hat{E}$ respectively. The existence of duality maps for stably finite complexes then implies that for a Hurewicz fibration $E$ with a finite-dimensional $C W$-complex $B$, a well-based cross-section, and fibres stably equivalent to a finite complex there is another such fibration $\hat{E}$ and an $n$ such that

$$
B \times S^{n} \stackrel{\mu}{\rightarrow} E \wedge{ }_{B} \hat{E}
$$

is a duality map. As in the usual theory, there is a duality map

$$
\hat{\mu}: E \wedge_{B} \hat{E} \rightarrow B \times S^{n} \text {. }
$$

Now if $F \rightarrow E \stackrel{p}{\rightarrow} B$ is a Hurewicz fibration, we denote by $\bar{E}$ the fibration $E$ disjoint union with a copy of $B$, which serves as the cross-section. The transfer map $\tau(f)$ is defined by

$$
\begin{gathered}
S^{n} \times B \stackrel{\mu}{\rightarrow} \bar{E} \wedge_{B} \hat{E} \stackrel{(1, f) \wedge 1}{\rightarrow} \bar{E} \wedge_{B} \bar{E} \wedge_{B} \hat{E} \stackrel{1 \wedge \hat{\mu}}{\rightarrow} \bar{E} \wedge_{B}\left(B \times S^{n}\right) \\
\downarrow \\
\left(B \times S^{n}\right) \wedge_{B} \bar{E}
\end{gathered}
$$

which gives $\tau(f): S^{n} \wedge(B / A) \rightarrow S^{n} \wedge\left(E / E_{A}\right)$ where $E_{A}=p^{-1}(A)$ and $f: E \rightarrow E$ is a fibre preserving map. All the properties of $\tau(f)$ go over to the case of stably finite fibres without changing a word of the proof.

Now a stably finite CW-complex has a very nice homological characterization. A theorem of Milnor states that a simply connected complex which has finitely generated integral homotopy has the homology type of a finite complex [W, Proposition 4.1].

Proposition 1. A CW-complex $X$ is stably finite if and only if $H_{*}(X ; \mathbf{Z})$ is finitely generated.

Thus the transfer is defined for fibrations with fibres $F$ such that $H_{*}(F ; \mathbf{Z})$ is finitely generated.

Transfer TheOrem. Let $F \stackrel{i}{\rightarrow} E \stackrel{p}{\rightarrow} B$ a Hurewicz fibration so that $H_{*}(F ; \mathbf{Z})$ is finitely generated, $F$ is a $C W$-complex, $B$ is a finite-dimensional $C W$-complex, $A \subset B$ is a subcomplex, and $f: E \rightarrow E$ is a map so that $p \circ f=p$. Then there exists an $S$-map $\tau(f): B / A \rightarrow E / E_{A}$ so that for singular homology $p_{*} \circ \tau(f)_{*}=\Lambda_{f^{\prime}}$ (multiplication by the Lefschetz number of $f^{\prime}=f \mid F: F \rightarrow F$ ). Also

(a) $\tau(f)^{*} \circ p^{*}=\Lambda_{f^{\prime}}$ on singular cohomology.

(b) For ring spectra $\tau(f)^{*}\left(p^{*}(\alpha) \cup \beta\right)=\alpha \cup\left(\tau(f)^{*}(\beta)\right)$ and $p_{*}\left(\tau(f)_{*}(x) \cap y\right)$ $=x \cap \tau(f)^{*}(y)$. 
A corollary of the transfer is the following result. Let $\omega: \Omega B \rightarrow F$ be the map arising from the fibration in the above theorem.

$\omega$ TheOREM. $\Lambda_{f^{\prime}}\{\omega\}=0 \in\{\Omega B, F\}$ where \{\} denotes the stable homotopy classes of maps.

Remark. This is the generalization of Theorem 1.1 of [BG]. Dold and Puppe can remove the finiteness hypothesis on $B$ in [DP; see Theorem 6.2]. Thus $B$ is a CW-complex, not necessarily finite dimensional.

3. Groups. In this section we consider the Transfer Theorem and $\omega$ Theorem in the context of group (co)homology.

There is a functor from groups and homomorphisms to spaces and maps so that for every group $G$ there is an Eilenberg-Mac Lane space $B_{G}$ and for every homomorphism $G \stackrel{\rho}{\rightarrow} G^{\prime}$ there is a map $r: B_{G} \rightarrow B_{G^{\prime}}$ which induces the corresponding homomorphism on fundamental group, i.e. $r_{*}: \pi_{1}\left(B_{G}\right) \cong G \stackrel{\rho_{*}}{\rightarrow} G^{\prime} \cong \pi_{1}\left(B_{G^{\prime}}\right)$.

From now on $\Gamma$ will always denote a constant group of coefficients. The homology (resp. cohomology) of a group $G$ with coefficients, $H_{*}(G ; \Gamma)$ (resp. $H^{*}(G ; \Gamma)$ ), is isomorphic to $H_{*}\left(B_{G} ; \Gamma\right)$ (resp. $\left.H^{*}\left(B_{G} ; \Gamma\right)\right)$.

Now a short exact sequence of groups

$$
1 \rightarrow H \stackrel{i}{\rightarrow} G \stackrel{\rho}{\rightarrow} K \rightarrow I
$$

gives rise to a fibration of classifying spaces

$$
B_{H} \rightarrow B_{G} \rightarrow B_{K} .
$$

If $H_{*}(H ; \mathbf{Z})$ is finitely generated, we may apply the transfer theorem to the fibration.

THEOREM 2. Let $1 \rightarrow H \stackrel{i}{\rightarrow} G \stackrel{\rho}{\rightarrow} K \rightarrow 1$ be an exact sequence. Let $H_{*}(H ; \mathbf{Z})$ be finitely generated. Let $f: G \rightarrow G$ be a homomorphism such that $\rho f=\rho$. Then,

(a) There exists a homomorphism $\tau_{*}: H_{*}(K ; \Gamma) \rightarrow H_{*}(G ; \Gamma)$ so that $\rho_{*} \circ \tau_{*}=\Lambda_{f^{\prime}}$, multiplication by the Lefschetz number of $f^{\prime}=f \mid H$.

(b) There is a homomorphism $\tau^{*}: H^{*}(G, \Gamma) \rightarrow H^{*}(K ; \Gamma)$ so that $\tau^{*} \rho^{*}=\Lambda_{f^{\prime}}$.

(c) $\tau^{*}\left(\rho^{*} \alpha \cup \beta\right)=\alpha \cup \tau^{*}(\beta)$ and $\rho_{*}\left(\tau_{*}(x) \cap y\right)=x \cap \tau^{*}(y)$.

REMARKS. (a) The Lefschetz number

$$
\Lambda_{f^{\prime}}=\sum_{i=0}^{\infty}(-1)^{i}\left(\operatorname{trace} f_{*}^{(i)}\right) .
$$

If $f$ is the identity homomorphism, then $\rho f=\rho$ and $\Lambda_{f^{\prime}}=\chi(H)$, the Euler-Poincare number.

(b) There are no conditions on $K$.

The map $\omega: \Omega B \rightarrow F$ factors through $F^{F}, 1 \stackrel{\omega_{x}}{\rightarrow} F$ where $F^{F}, 1$ is the space of self maps of $F$ homotopic to the identity and $\omega_{x}$ evaluates $f: F \rightarrow F$ at some point $x \in F$. In [G, Theorem II.2] if $F=B_{G}$, then $F^{F}, 1=B_{C}$ where $C$ is the center of $G$. Also $\omega_{x}$ : $B_{C} \rightarrow B_{G}$ induces the inclusion of $C$ into $F$. Thus the $\omega$ Theorem gives us the following: 
THEOREM 3. Suppose $\omega: H \rightarrow G$ is the inclusion homomorphism of a central subgroup $H$ into $G$. If $H_{*}(G ; \mathbf{Z})$ is finitely generated, then

$$
\chi(G) \omega_{*}=0: \tilde{H}_{*}(H ; \Gamma) \rightarrow \tilde{H}_{*}(G ; \Gamma)
$$

and

$$
\chi(G) \omega^{*}=0: \tilde{H}^{*}(G ; \Gamma) \rightarrow \tilde{H}^{*}(H ; \Gamma) .
$$

REMARK. In [G, Corollary 4.3], it was shown that if $\chi(G) \neq 0$ and $B_{G}$ is homotopy equivalent to a finite complex, then $G$ had trivial center. Stallings [Stg2] extended this to groups with finite free resolutions. But on the other hand, Baumslag, Dyer and Heller have shown that any abelian group can be the center of a group $G$ such that $\tilde{H}_{*}(G ; \mathbf{Z})=0,[\mathbf{B D H}$, Theorem 7.1].

Now [KT] have shown that for every space there is a group which has the same homology. [BDH] have shown that one may choose a group $G$ so that if the space were a finite complex then $B_{G}$ is homotopy equivalent to a finite complex.

\section{Applications.}

THEOREM 4. Suppose $H_{*}(H ; \mathbf{Z})$ is finitely generated where $H$ is a normal subgroup of $G$. If $H \subset[G, G]$, then $h^{\chi(H)} \in[H, G]$ for all $h \in H$.

Proof. Consider the Stallings-Stammbach exact sequence [Sts 1 , Theorem 2.1; Stch, c; Sj, Theorem 4.4],

$$
H_{2}(G) \stackrel{\rho_{*}}{\rightarrow} H_{2}(K) \stackrel{\partial}{\rightarrow} H /[H, G] \stackrel{i_{*}}{\rightarrow} H_{1}(G) \stackrel{\rho_{*}}{\rightarrow} H_{1}(K) \rightarrow 0
$$

where $\rho: G \rightarrow G / H=K$. Now $H_{1}(G ; \mathbf{Z})=G /[G, G]$. Since $H \subset[G, G]$ we see that $i_{*}=0$. Therefore $\partial$ is onto. Now there is a transfer homomorphism $\tau_{*}: H_{2}(K) \rightarrow$ $H_{2}(G)$ so that $\rho_{*} \tau_{*}=\chi(H)$. So $\chi(H)$ annihiliates every element of $H /[H, G]$.

TheOREM 5. Suppose $C$ is central in $H$. Suppose $H_{*}(H ; \mathbf{Z})$ is finitely generated. Then $c^{\chi(H)} \in[H, H]$ for all $c \in C$.

Proof. Let $\omega: C \rightarrow H$ be the inclusion. Then

$$
\chi(H) \omega_{*}=0: H_{1}(C) \cong C \rightarrow H /[H, H] \cong H_{1}(H) .
$$

REMARK. There is a curious relationship between the above two theorems. Note that $C$ is central in $H$ if and only if $C$ is normal and $[C, H]=1$. Now for any normal subgroup $H$ of $G$ we have the following series of normal subgroups in $H$.

$$
1 \subset[H, G] \subset H \cap[G, G] \subset H .
$$

The first theorem has the hypothesis that $H \cap[G, G]=H$ and the conclusion that $h^{x(H)} \in[H, G]$. The second theorem has the hypothesis that $[H, G]=1$ and the conclusion that $h^{\chi(G)} \in H \cap[G, G]$.

Recall that the lower central series of a group $G$ is a descending sequence of normal subgroups defined by $G_{1}=G, G_{\alpha+1}=\left[G_{\alpha}, G\right]$ and $G_{\beta}=\bigcap_{\alpha<\beta} G_{\alpha}$ for limit ordinals.

Corollary 6. Let $G$ be a group so that $H_{*}\left(G_{\alpha} ; \mathbf{Z}\right)$ is finitely generated and $\chi\left(G_{\alpha}\right)= \pm 1$. Then $G_{\alpha}=G_{\beta}$ for all $\beta \geqslant \alpha>1$. 
Proof. We only need to show that $G_{\alpha}=G_{\alpha+1}$. We apply Theorem 4 for the subgroup $H=G_{\alpha}$. Then $G_{\alpha} \subset G_{\alpha+1}$. Hence $G_{\alpha}=G_{\alpha+1}$.

REMARK. This corollary implies an interesting observation. It is a theorem of Magnus [M] that the intersection of the lower central series of a free group is the trivial subgroup 1. So a free group on two generators, $F$, has the property that $F_{\omega}=1$. But if $F$ is the commutator subgroup of some larger group $G$, then $G_{\omega}=F$. This follows since $\chi(F)=-1$. There do exist groups $G$ such that $G_{2}=F$. For example the knot group of the trefoil knot.

THEOREM 7. Let $\mathbf{Z}^{r} \stackrel{i}{\rightarrow} G \stackrel{\rho}{\rightarrow} K$ be a central extension where $\mathbf{Z}^{r}$ is the free abelian group of rank $r$. Suppose $\phi: G \rightarrow \mathbf{Z}^{r}$ is a homomorphism. Then there are transfers $\tau_{*}$ : $H_{*}(K ; \Gamma) \rightarrow H_{*}(G ; \Gamma)$ and $\tau^{*}: H^{*}(G ; \Gamma) \rightarrow H^{*}(K ; \Gamma)$ so that $\rho_{*} \circ \tau_{*}=\operatorname{det}(\phi \circ i)$ and $\tau^{*} \circ \rho^{*}=\operatorname{det}(\phi \circ i)$.

Proof. Suppose $f: \mathbf{Z}^{r} \rightarrow \mathbf{Z}^{r}$ is a homomorphism. Now $H_{*}\left(\mathbf{Z}^{r} ; \mathbf{Z}\right) \cong \Lambda\left(\mathbf{Z}^{r}\right)$, the exterior algebra on $\mathbf{Z}^{r}$. It is not difficult to see that the Lefschetz number of $f$ is $\Lambda_{f}=\operatorname{det}(I-f)$ where $I$ is the identity.

Now consider $\phi: G \rightarrow \mathbf{Z}^{r}$. We define $\Phi: G \rightarrow G$ by $\Phi(g)=g \cdot(\phi(g))^{-1}$. Since $\mathbf{Z}^{r}$ is in the center of $G, \Phi$ must be a homomorphism. Also note that $\rho \circ \Phi=\rho$ since $\phi(g)$ is in the kernel of $\rho$ for all $g$. Thus there exists transfers for homology and cohomology where the relevant number is the Lefschetz number of $\Phi \mid \mathbf{Z}^{r}: \mathbf{Z}^{r} \rightarrow \mathbf{Z}^{r}$. But $\Phi \mid \mathbf{Z}^{r}=I-\phi \circ i$ where we use additive notation. So

$$
\Lambda_{\Phi \mid \mathbf{Z}^{r}}=\operatorname{det}\left(I-\Phi \mid \mathbf{Z}^{r}\right)=\operatorname{det}(I-(I-\phi \circ i))=\operatorname{det}(\phi \circ i) .
$$

Theorem 2 gives the required transfer.

Central extensions are well understood. If $C \rightarrow G \rightarrow K$ is a central extension, it corresponds to an element $k_{G} \in H^{2}(K ; C)$. Conversely every element of $H^{2}(K ; C)$ corresponds to a central extension of $C$ by $K$. There is a cohomology exact sequence

$$
\begin{array}{r}
0 \rightarrow H^{1}(K ; C) \stackrel{\rho^{*}}{\rightarrow} H^{1}(G ; C) \stackrel{i^{*}}{\rightarrow} H^{1}(C ; C) \stackrel{\delta}{\rightarrow} H^{2}(K ; C) \\
\downarrow \rho^{*} \\
H^{2}(G ; C)
\end{array}
$$

Now $k_{G}=\delta(1)$ where

$$
1 \in H^{1}(C ; C) \cong \operatorname{Hom}\left(H_{1}(C ; \mathbf{Z}) ; C\right)=\operatorname{Hom}(C ; C)
$$

corresponds to the identity homomorphism 1: $C \rightarrow C$. In the case where $C=\mathbf{Z}^{r}$ we have the following result.

THEOREM 8. Let $k \in H^{2}\left(K ; \mathbf{Z}^{r}\right)$ have order $N$. Then the corresponding central extension $\mathbf{Z}^{r} \stackrel{i}{\rightarrow} G \stackrel{\rho}{\rightarrow}$ Kadmits transfers $\tau_{*}$ and $\tau^{*}$ such that $\rho_{*} \tau_{*}=N^{r}$ and $\tau^{*} \rho^{*}=N^{r}$. If $k$ has infinite order then the only possible transfers in integral cohomology satisfy $\tau^{*} \rho^{*}=0$.

Proof. Now $\rho^{*}(k)=0$, so if $k$ has infinite order and $\tau^{*}$ is a transfer so that $\tau^{*} \rho^{*}=N$, then $N k=\tau^{*} \rho^{*}(k)=0$. Hence $N=0$. 
If $N k=0$ we have $\delta(N \cdot 1)=0$. So $N \cdot 1 \in H^{1}\left(\mathbf{Z}^{r} ; \mathbf{Z}^{r}\right)$ is in the image of $i^{*}$ : $H^{1}\left(G ; \mathbf{Z}^{r}\right) \rightarrow H^{1}\left(\mathbf{Z}^{r}, \mathbf{Z}^{r}\right)$. That implies there is a $\phi^{\prime} \in \operatorname{Hom}\left(H_{1}(G, \mathbf{Z}) ; \mathbf{Z}^{r}\right)$ so that $\phi^{\prime} \circ i=N \cdot 1$.

Then $\phi: G \stackrel{h}{\rightarrow} H_{1}(G ; \mathbf{Z}) \stackrel{\phi^{\prime}}{\rightarrow} \mathbf{Z}^{r}$ satisfies $\phi \circ i=N: \mathbf{Z}^{r} \rightarrow \mathbf{Z}^{r}$. Here $h$ is the Hurewicz homomorphism which is onto. Now there are transfers $\tau_{*}$ and $\tau^{*}$ so that $\rho_{*} \tau_{*}=$ $\operatorname{det}(\phi \circ i)=\operatorname{det}(N)=N^{r}$ and similarly $\tau^{*} \rho^{*}=N^{r}$.

K. B. Lee has a proof of Theorem 8 which uses cochain arguments and avoids the exact sequence above.

Theorem 8 relates to work of Lawson and Yau. They study compact manifolds of nonpositive curvature in [LY]. If $M$ has nonpositive curvature, then $M$ must be a $K(\pi, 1)$. Now Lawson and Yau show that the center of $\pi$ must be a free abelian group of rank $k$ where $k$ is the dimension of the group of isometries of $M$. Thus one gets a central extension $0 \rightarrow \mathbf{Z}^{k} \rightarrow \pi_{1}(M) \rightarrow K \rightarrow 1$ where $K$ is the quotient of $\pi_{1}(M)=\pi$ and the center $\mathbf{Z}^{k}$.

Conner and Raymond [CR] (see p. 56, paragraph (f)) observe that the classifying element of this extension has finite order. Thus Theorem 8 applies here and there is a transfer for this extension. In particular, for rational coefficients, the homology of $M$ splits as a tensor product of $H^{*}(T, \mathbf{Q}) \cong \Lambda^{k} \mathbf{Q}$ and $H^{*}(K ; \mathbf{Q})$.

I would like to thank K. B. Lee for pointing out the connection of Theorem 8 and the above work.

\section{REFERENCES}

[BDH] G. Baumslag, E. Dyer and A. Heller, The topology of discrete groups, J. Pure Appl. Algebra 16 (1980), $1-47$.

[BG] J. C. Becker and D. H. Gottlieb, The transfer for fibrations and duality, Compositio Math. 33 (1976), 107-133.

[CR] P. E. Conner and F. Raymond, Deforming homotopy equivalences to homeomorphisms in aspherical manifolds, Bull. Amer. Math. Soc. 83 (1977), 36-85.

[DP] A. Dold and D. Puppe, Duality, trace, transfer (Proc. Internat. Conf. Geometric Topology), Polish Scientific Publishers, Warsaw, 1980.

[G] D. H. Gottlieb, A certain subgroup of the fundamental group, Amer. J. Math. 87 (1965), 840-856.

[KT] D. M. Kan and W. P. Thurston, Every connected space has the homologv of a $K(\pi, 1)$, Topology 15 (1976), 253-258.

[LY] H. Lawson and S.-T. Yau, Compact manifolds of nonpositive curvature, J. Differential Geom. 7 (1972), 211-228. MR 48 \# 12402

[M] W. Magnus, Beziehungen zwischen Gruppen und Idealen in einen speziellen Ring, Math. Ann. 111 (1935), 259-280.

[Sj] D. Sjerve, Group actions and generalized periodicity, Math. Z. 149 (1976), 1-11.

[Stg1] J. Stallings, Homologv and central series of groups, J. Algebra 2 (1965), 170-181.

[Stg2] __ Centerless groups-An algebraic formulation of Gottlieb's Theorem, Topology 4 (1965), 129-134.

[Stch] U. Stammach, Anwendungen der Homologie Theorie der Gruppen auf Zentralreihen und auf Invarianten von Prasentierungen, Math. Z. 94 (1966), 157-177.

[W] C. T. C. Wall, Finite conditions for CW-complexes, Ann. of Math. (2) 81 (1965), 56-69.

Department of Mathematics, Purdue University, West Lafayette, Indiana 47907 